\title{
Training the doctors of tomorrow to be leaders in quality improvement: the highs and lows of a new curricular initiative
}

\author{
Authors: Cindy Sethi, Ann McKee, Anne Wylie, Martha Martin, Danny Ruta and Louise Dubras
}

\begin{abstract}
Aims
In September 2016, the Medical School of King's College London, UK, introduced a new undergraduate medical curriculum with one of its themes being 'Population science and quality improvement' (QI). To develop understanding of QI methodology and skills within a clinical setting, every fourth year medical student participates in a module undertaking a QI project in a healthcare setting. The module was taught online on the virtual learning environment, with four 'face-to-face' tutorials with a project supervisor. The aim of this study was to assess the impact of the QI projects and student and supervisor evaluation of the first year of this module.
\end{abstract}

\section{Methods}

From September 2016 to March 2017, 420 medical students undertook a QI project, working in groups of 2-5. Projects were set in primary (9 projects) and secondary care ( 123 projects) in London and the south east of England. Electronic module evaluation was undertaken using a 5-point Likert scale (1 ranking as 'poor') and supervisors were interviewed for their feedback.

\section{Results}

All groups were able to identify a clinical problem, collect baseline data and design at least one Plan, Do, Study, Act cycle. Student success included one group being shortlisted for national Patient Safety Awards and five other projects received university funding for national/international conference presentation. Of a sample of 20 randomly selected projects, $27 \%$ showed no improvement, $9 \%$ achieved their improvement goal and $67 \%$ of projects demonstrated some improvement. Electronic module evaluation was undertaken (response rate $50 \%$ ). University-focused feedback was positive (allocation and organisation, 3.5/5), but students noted that while supportive, supervisors were not aware of what was expected from the module. Domains that scored highly were: the opportunity to work with peers (3.9), supervisors (4.1) and clinical teams (3.5).There was criticism of placements and travelling time. Supervisors' feedback indicated that the four scheduled tutorials were insufficient to adequately support students.

\section{Conclusion}

Bold new requirements for all students to undertake a QI project placed burdens on supervisors that impacted student experience. Despite some successes, it is clear that the delivery of large scale QI project work and the adoption of QI methodologies will take some time to be embedded, particularly with clinical faculty.

\section{Conflict of interest statement}

There are no conflicts of interest by any of the authors. 